\title{
A 54-Year-Old Man with Right Opaque Hemithorax and Worsening Shortness of Breath
}

\author{
Ankur Girdhar ${ }^{\mathrm{a}}$ Robert Zaiden ${ }^{\mathrm{b}}$ \\ Departments of ${ }^{\mathrm{a} I n t e r n a l}$ Medicine and ${ }^{\mathrm{b}}$ Medical Oncology, UF and Shands College of Medicine, Jacksonville, Fla., USA
}

\section{Case Report}

A 54-year-old male presented to the emergency department with complaints of acute-onset shortness of breath and right-sided pleuritic chest pain along with generalized weakness and an undocumented weight loss over the last month. He denied any cough, fever or sputum production. He was a chronic smoker with 40 pack-years of smoking. Family history was negative for any cancers.

On physical examination, the patient was alert and oriented but in respiratory distress using accessory muscles of respiration with an oxygen saturation of $95 \%$ on $28 \% \mathrm{~F}_{\mathrm{i}} \mathrm{O}_{2}$. Auscultation of the chest revealed right-sided, basilar decreased air entry with bronchial breathing in the inframammary and infra-axillary areas. A diffuse impaired/dull note was appreciated on the right side of the chest. The skin over the right hemithorax was warm and hard to touch. On abdominal examination, there was massive, palpable hepatomegaly which was tender. The remaining examination was unremarkable.

Chest X-ray (fig. 1) disclosed an almost complete opacification of the right hemithorax with the mediastinal silhouette being slightly displaced towards the left side. A CT scan (fig. 2) of the chest showed lobular pleural and parenchymal masses in the right lung, and right hilar and mediastinal adenopathy with a few scattered air bronchograms. Ultrasound of the abdomen revealed an enlarged liver with two mass lesions with sizes of 2-2.4 and $1.7-2 \mathrm{~cm}$.

What is your diagnosis?
Fig. 1. Several expansile lesions in the right ribs are again noted. There appears to be some destruction of the right first rib from the adjacent mass. There is compression of the right pulmonary artery, left atrium and right mainstem bronchus. The right lung demonstrates a minimal amount of aerated lung from the compression due to the large mass occupying the right hemithorax.

Fig. 2. Near complete opacification of the right hemithorax corresponding to a large heterogeneous mass on chest CT.
1

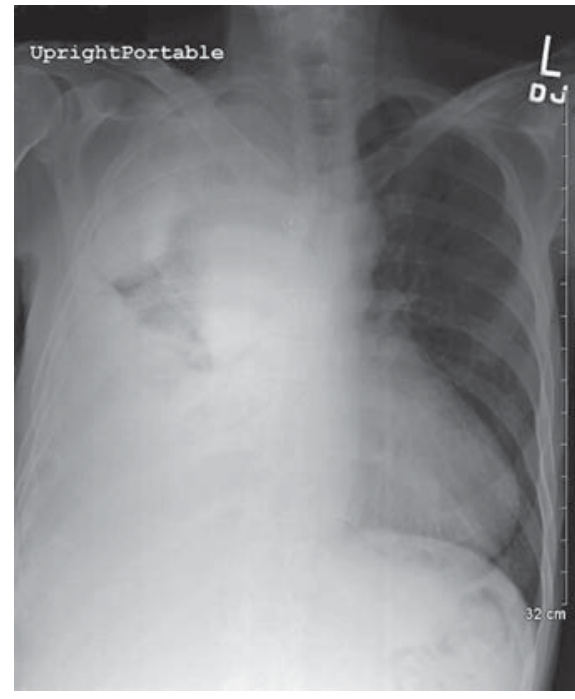

2

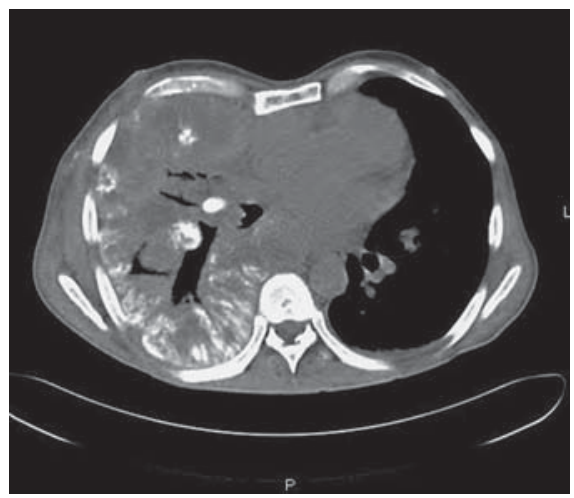

\section{KARGER}

Fax +4161306 1234 E-Mail karger@karger.ch www.karger.com
(C) 2009 S. Karger AG, Basel 0025-7931/10/0792-0157\$26.00/0

Accessible online at:

www.karger.com/res
Ankur Girdhar, MD

Department of Internal Medicine, UF and Shands College of Medicine 655 8th Street West

Jacksonville, FL 32209 (USA)

Tel. +1 904244 3094, Fax +1 904244 3634, E-Mail ankurgirdhar@yahoo.com 


\section{Diagnosis: Myxofibrosarcoma of the Lung}

A CT-guided fine-needle aspiration of the right-sided opacity revealed a mixed-size spindle-cell sarcoma with myxoid fibrosarcoma. The cells were negative for cytokeratin (AE1/AE3 and CAM 5.2), CEA, BER-EP, TTF-1, D2-40, calretinin, caldesmon, MOC-31, CD34 and S100, and positive for vimentin and focally positive for smooth muscle actin. The morphology of the malignant cells in the sample and the immunophenotype were diagnostic of sarcoma and most consistent with myxofibrosarcoma (MFS).

\section{Discussion}

MFS, formerly known as a myxoid variant of malignant fibrous histiocytoma [1], is one of the most common sarcomas in the extremities of elderly people and occurs more frequently in the dermal and subcutaneous tissues than deeper soft tissues [2]. Distant metastases are more common in locally recurrent low-grade MFS [3].

Primary lung sarcomas are uncommon histological types of primary lung cancer (accounting for $0.013-0.4 \%$ of all lung malignancies $[4,5])$ and present a wide spectrum of clinical behavior [6]. It is estimated that 1 in 500 primary lung malignancies is a sarcoma [7]. With less than 250 patients reported in the English medical literature, very little is known about the clinical behavior and treatment of these tumors. Since the advent of immunochemistry, much progress has been accomplished in the characterization and nosological classification of soft-tissue neoplasms. A European study performed to assess long-term survival in patients found out that in all the patients with a diagnosis of lung sarcoma, the most common histological type was malignant fibrous histiocytoma [6].

Primary pulmonary sarcomas must be distinguished from the more frequent occurrence of sarcomas metastatic to the lung, primary pulmonary sarcomatoid carcinomas and diffuse malignant mesotheliomas involving the lung. The expanded differential diagnosis might include other sarcomas (epithelioid hemangioendotheliomas, angiosarcomas, synovial sarcomas, liposarcomas, myogenic or neurogenic tumors, and malignant solitary fibrous tumors) and melanomas [8]. Some of the rare tumors that may mimic sarcomas would be pulmonary rhabdomyosarcomas and pleural solitary localized fibrous tumors [9]. Soft-tissue sarcomas constitute a heterogeneous group of neoplasms that frequently share similar morphologic features, thereby complicating the establishment of a correct diagnosis. As an ancillary technique, immunohistochemistry is an invaluable tool that provides excellent information assisting the pathologist in establishing a precise diagnosis, as well as providing relevant prognostic and therapeutic information [10].

In our patient, there was no past history of a soft-tissue lesion or surgical intervention for a benign lesion. A thorough physical examination failed to reveal any suspicious pathology anywhere else in the body. In view of the primary site other than the lung being unlikely, this was determined to be a primary fibrosarcoma of the lung.

Surgical resection, if complete, is curative for small, well-differentiated primary lung sarcomas $[2,6]$. Poorly differentiated tumors are less curable, but resection may provide palliation and should be considered. Unresectable or recurrent sarcomas may be treated with radiation therapy or chemotherapy, although responses are usually brief and survival is $<1$ year [11].

After discussing aggressive chemotherapy versus tumor chemoembolization, the patient chose a trial of chemoembolization to attempt tumor size reduction. In order to provide him with some comfort and to reduce the pressure symptoms of the lung mass, chemoembolization of intercostal arteries using Adriamycin was done. After undergoing the chemoembolization procedure, he reported some symptomatic benefit but radiologically there was not much improvement in the size of the tumor. The patient subsequently expired secondary to respiratory failure.

\section{Conclusion}

MFS, a common sarcomatoid tumor in the extremities of the elderly, is rarely found in the lung. MFS of the lung occurs mostly as a metastasis from a primary tumor elsewhere in the body. Immunohistochemistry is the key to histological diagnosis of this tumor. At this time, knowledge about clinical behavior and treatment of this tumor is limited. 


\section{References}

1 Weiss SW, Enzinger FM: Myxoid variant of malignant fibrous histiocytoma. Cancer 1977;39:1672-1685.

2 Wada T, Hasegawa T, Nagoya S, et al: Myxofibrosarcoma with an infiltrative growth pattern. Jpn J Clin Oncol 2000;30:458-462.

3 Huang H-Y, Lal P, Qin J, Brennan MF, Antonescu CR: Low-grade myxofibrosarcoma: a clinicopathologic analysis of 49 cases treated at a single institution with simultaneous assessment of the efficacy of 3-tier and 4-tier grading systems. Hum Pathol 2004;35:612621.
4 Guccion JG, Rosen SH: Bronchopulmonary leiomyosarcoma and fibrosarcoma: a study of 32 cases and review of the literature. Cancer 1972;30:836-847.

5 Martini N, Hajdu SI, Beattie EJ: Primary sarcoma of the lung. J Thorac Cardiovasc Surg 1971;61:33-38.

6 Magne N, Porsin B, Pivot X, et al: Primary lung sarcomas: long survivors obtained with iterative complete surgery. Lung Cancer 2001;31:241-245.

7 Iverson L: Bronchopulmonary sarcoma. J Thorac Surg 1954;27:130-148.
8 Litzky LA: Pulmonary sarcomatous tumors. Arch Pathol Lab Med 2008;132:1104-1117.

9 Kulinna-Cosentini C, Brunner C, Klepetko W, Dekan G, Bankier A: Incidental discovery of a large thoracic mass in a 65-year-old dentist. Respiration 2008;75:109-112.

10 Fos SN, Bosch AL: Immunohistochemistry of soft tissue sarcomas. Pathol Case Rev 2008; 13:45-50.

11 Regnard JF, Icard P, Guibert L, et al: Prognostic factors and results after surgical treatment of primary sarcomas of the lung. Ann Thorac Surg 1999;68:227-231. 